\title{
Effect of Lactylate and Bacillus subtilis on Growth Performance, Peripheral Blood Cell Profile, and Gut Microbiota of Nursery Pigs
}

\author{
Xiaofan Wang ${ }^{1}$, Tsungcheng Tsai ${ }^{1}{ }^{\oplus}$, Xiaoyuan Wei ${ }^{1} \mathbb{C}$, Bin Zuo ${ }^{1}$, Ellen Davis ${ }^{2}$, Tom Rehberger ${ }^{2}$, \\ Samantha Hernandez ${ }^{2}$, Evelien J.M. Jochems ${ }^{3}{ }^{\oplus}$, Charles V. Maxwell ${ }^{1}$ and Jiangchao Zhao ${ }^{1, *}$ \\ 1 Department of Animal Science, Division of Agriculture, University of Arkansas, Fayetteville, AR 72701, USA; \\ xxw033@uark.edu (X.W.); ttsai@uark.edu (T.T.); xw010@uark.edu (X.W.); binzuo@uark.edu (B.Z.); \\ cmaxwell@uark.edu (C.V.M.) \\ 2 Arm \& Hammer Animal and Food Production, Church \& Dwight, Inc., Waukesha, WI 53186, USA; \\ Ellen.Davis@churchdwight.com (E.D.); Tom.Rehberger@churchdwight.com (T.R.); \\ Samantha.Hernandez@churchdwight.com (S.H.) \\ 3 Corbion, Arkelsedijk 46, 4206 AC Gorinchem, The Netherlands; e.jochems@corbion.com \\ * Correspondence: jzhao77@uark.edu
}

Citation: Wang, X.; Tsai, T.; Wei, X.; Zuo, B.; Davis, E.; Rehberger, T.; Hernandez, S.; Jochems, E.J.M.; Maxwell, C.V.; Zhao, J. Effect of Lactylate and Bacillus subtilis on Growth Performance, Peripheral Blood Cell Profile, and Gut Microbiota of Nursery Pigs. Microorganisms 2021, 9, 803. https://doi.org/10.3390/ microorganisms 9040803

Academic Editors: Jana Seifert and Haruki Kitazawa

Received: 8 March 2021

Accepted: 6 April 2021

Published: 10 April 2021

Publisher's Note: MDPI stays neutral with regard to jurisdictional claims in published maps and institutional affiliations.

Copyright: (c) 2021 by the authors. Licensee MDPI, Basel, Switzerland. This article is an open access article distributed under the terms and conditions of the Creative Commons Attribution (CC BY) license (https:/ / creativecommons.org/licenses/by/ $4.0 /)$.

\begin{abstract}
To evaluate the effects of lactylate and Bacillus subtilis on growth performance, complete blood cell count, and microbial changes, 264 weaning pigs were assigned to four treatments (1) control (Con) basal diets that met the nutrient requirement for each phase, (2) $0.2 \%$ lactylate (LA), (3) $0.05 \%$ Bacillus subtilis strains mixtures (BM), or (4) the combination of LA and BM (LA+BM) added to the control basal diet at their respective inclusion rates in each of the three phases. Dietary lactylate tended to increase weight gain, significantly increased feed intake, and reduced fecal total E. coli and enterotoxigenic E. coli counts during Phase 1. Pigs fed Bacillus subtilis had a greater gain to feed ratio (G:F) during Phases 1 and 2. Pigs fed lactylate had an increased peripheral absolute neutrophil count on D14 but a decreased eosinophil percentage. Pigs fed Bacillus subtilis had an elevated peripheral total white blood cell count at study completion. The addition of lactylate increased microbiota richness, reduced E. coli, and increased Prevotella, Christensenellaceae, and Succinivibrio. Bacillus subtilis supplementation-enriched f_Ruminococcaceae_unclassified and S24-7_unclassified had positive relationships with feed efficiency. Collectively, these findings suggested that lactylate can be added to diets to balance gut microbiota and improve growth performance during the early postweaning period. The combination of lactylate and Bacillus subtilis strains exerted a synergic effect on the growth performance of nursery pigs.
\end{abstract}

Keywords: lactylate; Bacillus subtilis; growth performance microbiome; pig

\section{Introduction}

Postweaning is the most challenging period of life for swine because environmental changes and diet formulations force the reprogramming of the digestive system and the gut microbiota community [1,2]. Under this circumstance, the ecological balance of gut microbiota can be severely disturbed, and piglets can become very susceptible to pathogens that cause diarrhea, morbidity, and mortality [3]. Health during the early nursery period has a long-term impact on growth rates and feed efficiency, all of which have significant economic consequences for swine producers [4]. Maintaining gut homeostasis is considered the primary means to mitigate the detriments associated with weaning. Most strategies for addressing weaning stressors are gut-microbiota-associated [5,6]. This is because the gut microbiota have a multitude of functions in host defense, such as nutrient metabolism, energy replenishment, and establishing gastrointestinal barrier integrity $[7,8]$.

Dietary energy contributes to the greatest proportion of feed cost in the swine industry $[9,10]$. In addition to carbohydrates and fat from grain, dietary fat and oils are 
major energy sources. Fat digestion begins with emulsification by bile to form micelles, which increases the accessibility to pancreatic lipase [11]. The digestive system of weaning piglets is incompetent in processing dietary fat from solid diets [12]; hence, supplementing exogenous emulsifiers can promote fat absorption and retention. Lactylates (LA) are esterified lactic acid and fatty acid compounds that have been approved by the Food and Drug Administration for use as emulsifiers in food production [13]. Recently, growing evidence of its benefits on animal husbandry has been described [14]. Several studies have demonstrated the beneficial effects of sodium stearoyl-2-lactylate (SSL) on growth performance and nutrient digestibility in young pigs and producing sows $[15,16]$. Similar responses were also observed in broilers when SSL (0.05-0.1\%) was supplemented in a common diet [17] or an energy-reduced diet [18].

Direct-fed microbiota (DFM) are beneficial microorganisms that are commonly used in diet interventions to improve livestock health. There are three major types of DFM in the swine industry: Lactobacillus, yeast, and Bacillus. Certain Bacillus sp., the most preferred DFM, are spore-forming aerobes [19] with thermostable and low-pH-resistant characteristics. They can be easily mixed into solid feed and survive the digestive system. These Bacillus sp. have been determined to possess beneficial probiotic capabilities on growth performance and disease resilience in pigs via gut microbiota manipulation [20]. For instance, Hu et al. found that a Bacillus KN-42-supplemented diet not only improved growth performance but also reduced the incidence of diarrhea in weaned pigs, perhaps by stimulating Lactobacillus and suppressing E. coli $[20,21]$. Other research institutes unveiled the defensive mechanisms of beneficial Bacillus against enterotoxigenic E. coli (ETEC) in young piglets by noting that piglets that received Bacillus probiotics had enhanced goblet cell function and gut integrity, which ameliorated ETEC-induced enteritis [22,23].

The purpose of the current project is to examine the effects of active lactylate (LA) and Bacillus subtilis strain mixtures (BM) on growth performance, peripheral blood cell counts, pathogen count, and the gut microbiome in pigs. By profiling the gut microbiota in each treatment, we discuss the potential mechanisms of how the driven microbiota community influences the growth performance of nursery pigs and use a machine learning method to reveal taxa that best represent the impact of the treatments.

\section{Materials and Methods}

Pigs were managed according to the University of Arkansas Institutional Animal Care and Use Committee guideline (IACUC\# 21038).

\subsection{Animals, Experimental Design, and Dietary Treatments}

A total of 264 weanling pigs (PIC1050 $\times$ DNA600) were selected at weaning (Day $21 \pm 2$ ) and transferred to a conventional nursery facility at the University of Arkansas Swine Research Unit. Pigs were blocked by initial body weight into 11 groups within each sex and allotted to 1 of 44 plastic floor pens. Pens then were randomly assigned to one of four dietary treatments in a $2 \times 2$ factorial arrangement, including (1) control (Con) basal diets that met the nutrient requirement for each phase (Table S1), (2) $0.2 \%$ lactylate (LA), (3) $0.05 \%$ Bacillus subtilis strains mixtures (BM), or (4) the combination of LA and BM $(\mathrm{LA}+\mathrm{BM})$, added to the control basal diet at their respective inclusion rates in each of the three phases. The types of active lactylate (ALOAPUR ${ }^{\circledR}$, Corbion, The Netherlands) used in this trial were ester compounds made from lactic acid with lauric acid and myristic acid, using diatomaceous earth material as the carrier. Bacillus subtilis strains (B. $747+$ B. 1999) were supplied by Arm and Hammer Animal and Food Production (Certillus ${ }^{\mathrm{TM}}$, Waukesha, WI, USA). All diets were devoid of feed antibiotics and pharmaceutical levels of zinc and copper.

Each pen housed three male and three female pigs, and they remained on the same treatment throughout the study period. Pigs were fed a three-phase dietary regimen with a 14-day duration per phase (Phase 1: D0-14; Phase 2: D14-28; Phase 3: D28-42). Each pen (size: $1.5 \times 1.2 \mathrm{~m}^{2}$ ) was equipped with a two-hole feeder and a cup waterer, and 
pigs had free access to feed and water during the entire trial. The nursery facility was a totally confined building, and the ambient temperature was programmed to start at $29{ }^{\circ} \mathrm{C}$ at weaning and declined sequentially to $25^{\circ} \mathrm{C}$ by the end of the trial. Fluorescent lighting was provided $24 \mathrm{~h}$ per day during the entire study.

\subsection{Growth Performance, Sample Collections, and Processing}

Individual pig body weight (BW) was measured at weaning and the end of each phase to calculate average daily gain (ADG). Feed intake was recorded throughout the trial to determine the average daily feed intake (ADFI) during each phase. Feed efficiency (FE or G:F) was defined as the amount of body weight gain from a given pen divided by the feed consumed during a period. Body weight, feed added, and leftover feed was weighed using a customized weight scale (IQ Plus ${ }^{\circledR} 390 D C$, Rice Lake Weighing System, Rice Lake, WI, USA). A 50-lbs weight block was used to validate the reading of the scale prior to and post weighing. Fresh feces, rectal swabs, and blood samples were collected from a median body weight barrow in each pen at weaning, and the same pigs were sampled during the entire trial.

Fecal samples were assayed for Clostridium, E. coli, and Streptococcus suis counts at the Arm and Hammer Animal and Food Production Laboratory (Waukesha, WI. USA), following procedures outlined in the Bacteriological Analytical Manual (8th Ed., 1998). Briefly, fecal samples were diluted 1:10 in $0.1 \%$ peptone, masticated, and plated onto selective media. Total Clostridium and C. perfringens were isolated using Perfringens agar base (Oxoid Ltd., Cambridge, UK) with tryptose sulfite cycloserine (TSC) supplement (Oxoid Ltd.). Clostridium perfringens isolates were confirmed using multiplex PCR for the detection of alpha toxin [24]. Escherichia coli were enumerated on CHROMagar ${ }^{\mathrm{TM}}$ (CHROMagar, Paris, France). Isolates were confirmed as enterotoxigenic E. coli (ETEC) using multiplex PCR methods adapted from Stacy-Philips et al. [25] and Casey and Bosworth [26]. Streptococcus was isolated and enumerated on Todd-Hewitt broth (THB), and S. suis was confirmed by PCR using the primer set developed by Okwumabua et al. [27].

Plasma lactylates were analyzed via liquid chromatography using an Acquity UPLC system (Waters, Milford, MA, USA) with an analytical column (Acquity UPLC BEH C18, $50 \times 2.1 \mathrm{~mm}$ i.d., $\mathrm{dp}=1.7 \mu \mathrm{m}$ (Waters)) and an Inline filter (ASSY frit, 2.1. $\mathrm{mm}$ id., $0.2 \mu \mathrm{m}$ (Waters)) at a column oven temperature of $40 \pm 1{ }^{\circ} \mathrm{C}$.

The rectal swabs were frozen immediately at $-80^{\circ} \mathrm{C}$ until microbiome analysis. Blood samples were drawn via jugular vena and collected into K2EDTA-coated vacutainer tubes for plasma lactylate evaluation (Charles River Laboratories, 's-Hertogenbosch, The Netherlands) and complete blood count (CBC) determination using a Hemavet 950 (Drew Scientific, Miami Lakes, FL, USA).

\section{3. $16 S$ rDNA Amplicon Sequencing}

A volume of $200 \mu \mathrm{L}$ of fecal suspension from each rectal swab $(n=172)$ was used for 165 rDNA sequencing, as previously described [28]. Briefly, DNA extractions were performed with the PowerLyzer PowerSoil DNA Isolation Kit (Qiagen, Germantown, MD, USA), following the manufacturer's protocol. Extracted DNA was subjected to quantity and quality determination by a NanoDrop spectrophotometer (Thermo Fisher Scientific, Wilmington, DE, USA), followed by library construction and 16S rDNA amplicon sequencing. Bacterial 16S rDNA V4 amplicons were generated by polymerase chain reaction (PCR) using primers (F: 5'- GTGCCAGCMGCCGCGGTAA - $3^{\prime}$ and R: 5' - GGACTACHVGGGTWTCTAAT $-3^{\prime}$ ) with the attachment of eight base pairs of the barcoded index and Illumina flow cell adapters. Accuprime Pfx Supermix (Invitrogen, Carlsbad, CA, USA) was used as the PCR reagent because of its high fidelity amplification. PCR products were cleaned and normalized by a SequalPrep Normalization Plate Kit (Invitrogen, Carlsbad, CA, USA) to balance DNA densities between samples. A Qubit 3 fluorometer (Thermo Fisher Scientific, Waltham, MA, USA) was used to validate the normalization step, and then purified DNAs were pooled in equal volume. The DNA pool was further subjected 
to quality and quantity determination with an Agilent Bioanalyzer 2100 (Agilent, Santa Clara, CA, USA) and quantitative RT-PCR, respectively. For sequencing, Illumina MiSeq $2 \times 250$-bp paired-end sequencing (MiSeq Reagent Kit v2, 500 cycles, 20\% PhiX) was used. Before loading into the cartridge, DNA pools were denatured with freshly made $0.2 \mathrm{~N}$ $\mathrm{NaOH}$ solution and then diluted with HT1 buffer. $\mathrm{NaOH}$-denatured Phix was incorporated into the final pool at $20 \%$ to increase nucleotide diversity at each cycle to improve the reading. Negative control and mock communities (ZymoBIOMICS ${ }^{\mathrm{TM}}$ Microbial Community Standard; Zymo, Irvine, CA, USA) were included as two samples for future quality evaluation.

\subsection{Statistical Analysis for Growth Performance, Complete Blood Cell Count, and Microbial Counts}

Growth performance measures (BW, ADG, ADFI, and G:F) and complete blood cell count $(\mathrm{CBC})$ data were analyzed by ANOVA using the mixed procedure of SAS 9.3 (SAS Institute, Inc., Cary, NC, USA) in a randomized complete block design, with a $2 \times 2$ factorial dietary treatment arrangement. The pens served as the experimental unit. The model included a block as the random effect, with a $0.2 \%$ lactylate and $0.05 \%$ Bacillus subtilis mixture and its interaction as the fixed effects. Student's $t$-test was used in multiple comparisons to test the significance. The microbial count data were analyzed by diet phase using the GLM procedure of JMP ${ }^{\circledR}$ version 15.0.0 (SAS Institute, Inc., Cary, NC, USA, 1989-2019). A $p$-value $<0.05$ was considered significant, and $0.05<p$-value $<0.10$ was considered a statistical trend.

\subsection{Microbiota Data Analysis}

Raw reads (forward and reverse for each sample) generated by Miseq were uploaded into the QIIME2 platform (v. 2.4). Sequencing reads analysis followed general steps, including paired reads joining, quality filtering, denoising (Deblur in QIIME2), classification (Greengenes reference database; version 13-8), rarefying (5982), and bacterial units clustering $(100 \%)$. Alpha (Shannon index, observed features) and beta diversity (Bray-Curtis) were evaluated for microbial diversity and distances between subjects, respectively. Differentially represented bacterial members between groups were determined using the online software LEfSe (https: / / huttenhower.sph.harvard.edu/galaxy/; access date: 1 December 2020). Classification and regression-based random forest models were developed to identify treatment- or growth-performance-associated bacterial members, respectively. Mothur software v. 1.39 .5 (corr.axes) was used to calculate the coordinates for each significantly affected blood component. R software was used for data visualization.

This sequencing and data analysis pipeline yielded high-quality reads, as suggested by the clustered mock communities' consistent relative abundances of each species between different Miseq runs.

\section{Results}

No significant clinical symptoms of diseases were observed throughout the study except for minor postweaning scours. Scours were not associated with any of the treatments. Two pigs, one each from Treatments 2 and 4, were removed from the trial due to illness. Plasma C12 and C14 lactylate measurements demonstrated that the active lactylates used in this trial were absorbed from the lumen of the GI tract and translocated into systemic circulation. As shown in Table S2, the plasma concentrations of both C12 and C14 lactylates were below the detectable limit $(<5 \mathrm{ng} / \mathrm{mL})$ in control pigs, while the levels of $\mathrm{C} 12$ and C14 lactylates were detected at around 125 and $15 \mathrm{ng} / \mathrm{mL}$, respectively, in pigs fed a diet supplemented with lactylates throughout the study (Table S2). In addition, plasma C12 and C14 lactylates in pigs fed $0.2 \%$ LA were higher at the end of Phases 2 and 3 than Phase 1. However, it gradually declined from Phases 1 to 3 in pigs fed both LA and BM. 


\subsection{Growth Performance, Complete Blood Count, and Microbial Counts}

The addition of lactylates to the diet tended to increase average daily gain (ADG) compared to pigs fed diets devoid of lactylates $(0.088 \mathrm{vs.} 0.075 \mathrm{~kg} / \mathrm{d}$; LA: $p=0.07$; Table S3 and Figure 1a,b). No statistically significant differences in ADG were observed in pigs fed the Bacillus subtilis mixture. Pigs fed diets containing lactylates alone had an increased average daily feed intake (ADFI) during Phase 1 but not when fed in combination with BM (Table S4, LA $\times$ BM interaction $p=0.03$ ). Pigs fed the Bacillus subtilis mixture tended to have a greater gain-to-feed ratio (G:F) during Phase 1 (BM: $p=0.08$; Figure 1c,d) and Phase 2 (BM: $p=0.05$; Figure 1e,f) than pigs fed the Bacillus-subtilis-free diets. The improvement in G:F in pigs fed Bacillus subtilis was mainly driven by pigs fed diets supplemented with both lactylate and Bacillus subtilis mixtures (Phases 1 and 2, LA $\times$ BM interaction, $p=0.04$; Table S4). Although there were no significant treatment effects on body weight $(p>0.10$ for all three phases), pigs fed diets containing both lactylates and Bacillus subtilis were $0.26,0.63$, and $0.85 \mathrm{~kg}$ heavier than the control groups at the end of Phases 1, 2 and 3, respectively (Table $\mathrm{S} 4$ ).

Both dietary lactylates and the Bacillus subtilis mixtures caused noticeable effects on peripheral blood cell counts. Pigs fed lactylates had a higher absolute neutrophil (NE) count at the end of Phase 1 (D14) compared to pigs fed diets devoid of LA; however, the NE count decreased over time to values lower in pigs fed LA on D28 and D42 than in pigs fed diets devoid of LA (Table S5, LA $\times$ day $p=0.04$ ). The percentage of the eosinophil in peripheral blood decreased at a greater magnitude in pigs fed with LA compared to their counterparts on D14. The percentage of monocytes over total white blood cell count increased over time from the end of Phase 1 to study completion in pigs fed LA, whereas there was a lack of time response in pigs fed LA-free diets (Table S5, LA $\times$ day $p<0.01$ ). An age-associated response in peripheral total white blood cell counts differentiated BM from BM-free diets. Absolute total white blood cell counts increased from weaning to the end of Phase 2 (d28) in both pigs fed BM and BM-free diets. While the level of peripheral total white blood cells remained similar in pigs fed $\mathrm{BM}$ at the end of the trial, a decline was observed in pigs fed BM-free diets (Table S6, BM $\times$ day $p<0.01$ ). This observation on total white blood cell counts was mainly driven by absolute lymphocyte count (Table S6, BM $\times$ day $p<0.01$ ). An expansion of absolute neutrophil count occurred in both BM and BM-free treatments from Phases 1 to 2. However, the magnitude of expansion was lower in BM than BM-free treatments (Table S6, BM $\times$ day $p=0.06$ ). Both red blood cell counts and the percentage of hematocrits decreased from weaning to the end of Phase 2 in pigs fed BM-free diets, but the levels were relatively stable in pigs fed BM-supplemented diets across age (Table S6, $\mathrm{BM} \times$ day $p=0.07)$. Pigs fed BM had the lowest hemoglobin concentrations, whereas the diets containing lactylate alone and in combination with $\mathrm{BM}$ had similar hemoglobin levels compared to the control pigs ( $\mathrm{LA} \times \mathrm{BM}$ interaction, $p=0.02$; Figure $1 \mathrm{~g}$ ).

Dietary lactylates reduced the bacterial cell counts of major pathogens during the early nursery stage, which coincided with weight gain improvement. At the beginning of the study, the Streptococcus suis cell count in feces in pigs fed only LA was similar to those pigs fed control diets. While pigs fed BM alone had similar counts of Strep suis, a lower count was showed in pigs assigned to the combination of BM and LA (BM $\times$ LA interaction, $p=0.05)$, but this pattern disappeared after that (Table S7). Supplementing LA in the diet reduced $(p<0.05)$ fecal total E. coli $\left(6.62 \pm 0.29\right.$ vs. $\left.7.72 \pm 0.30 \log _{10} \mathrm{CFU} / \mathrm{g} ; p=0.01\right)$ and enterotoxigenic E. coli (6.62 \pm 0.30 vs. $\left.7.72 \pm 0.29 \log _{10} \mathrm{CFU} / \mathrm{g} ; p=0.01\right)$ counts at the end of Phase 1 compared to pigs fed diets devoid of LA (Figure 1h). At the end of Phase 3, pigs fed diets containing LA had greater $(p=0.02)$ total Clostridium $(6.31 \pm 0.23$ vs. $5.11 \pm 0.23$ $\log _{10}$ CFU/g; LA: $p<0.01$ ) and C. perfringens type A (5.93 \pm 0.27 vs. $5.04 \pm 0.26 \log _{10}$ $\mathrm{CFU} / \mathrm{g}$; LA: $p=0.02$; Figure 1i) presence in feces compared to pigs fed diets without LA. 
a

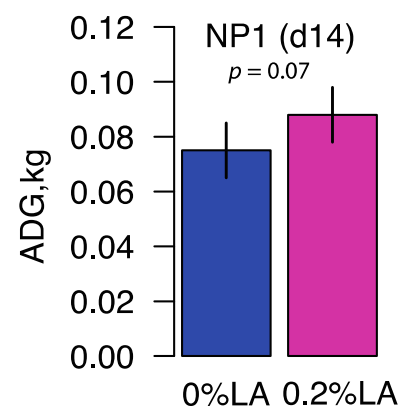

C
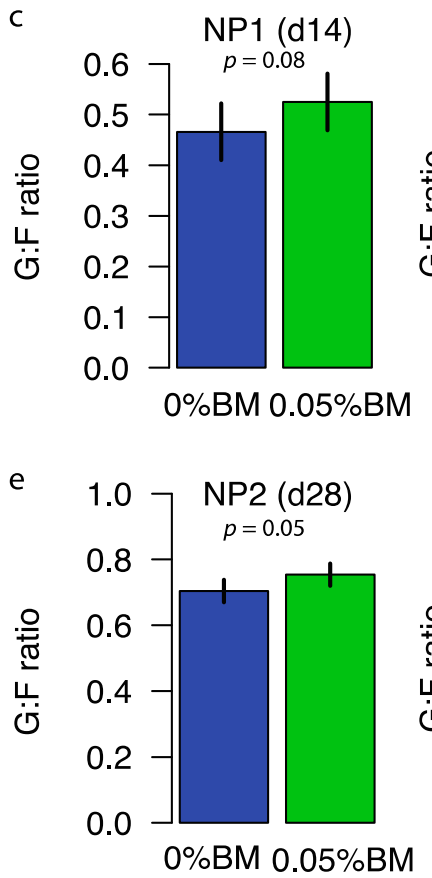

b

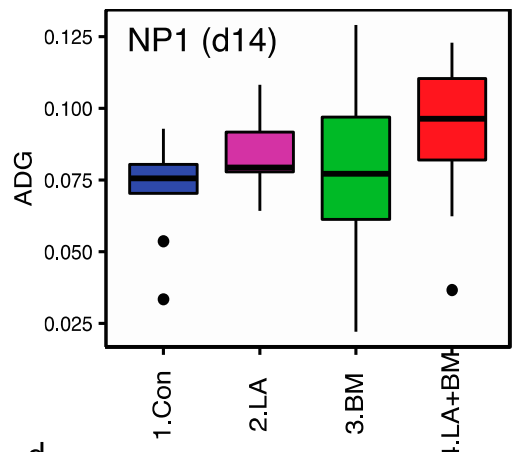

d
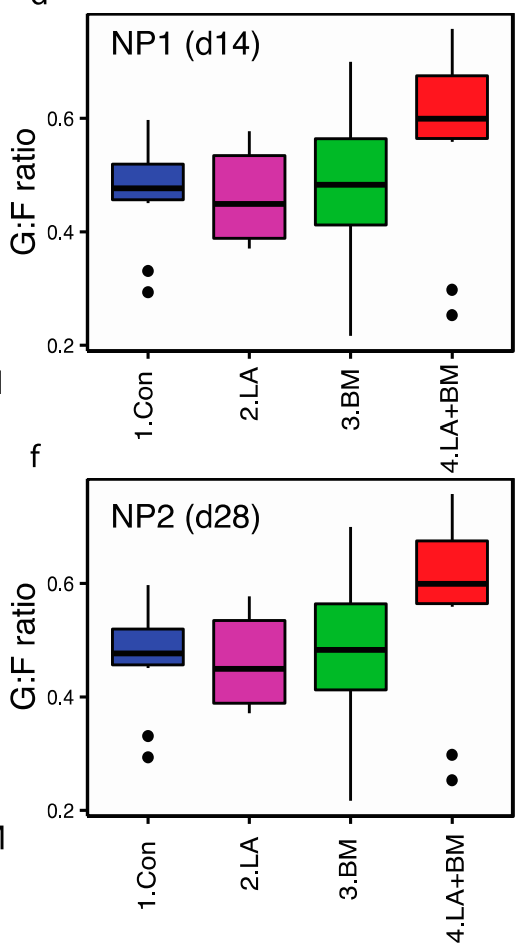

$\mathrm{g}$

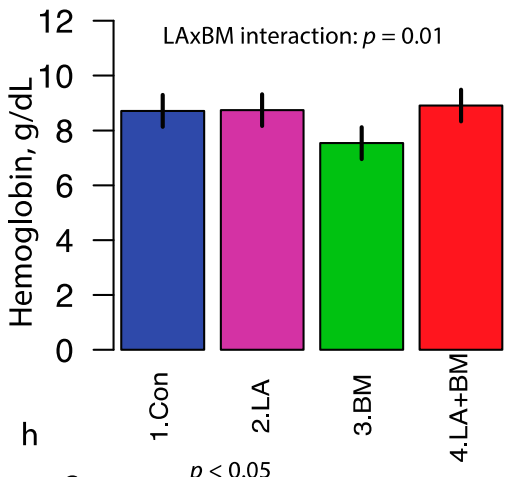

i

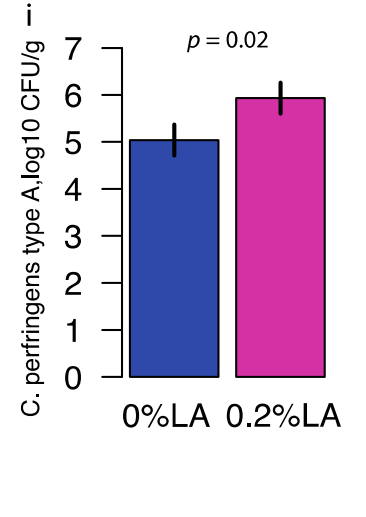

Figure 1. Effects of lactylate (LA) and Bacillus subtilis (BM) on average daily gain (ADG) at the end of nursery phase 1 (NP1) $(\mathbf{a}, \mathbf{b})$; feed efficiency at the end of NP1 (c,d) and NP2 (e,f); hemoglobin (g); E. coli counts at the end of NP1 (h); C. perfringens type A counts at the end of NP3 (i). A total of 264 pigs of weaning age were stratified by initial body weight (BW) and gender and assigned to a $2 \times 2$ factorial arrangement trial. The four dietary treatments were (1) control (Con), (2) $0.2 \%$ lactylate (LA), (3) $0.05 \%$ Bacillus subtilis strain mixtures (BM), or (4) the combination of LA and BM (LA + BM), added to the control basal diet at their respective inclusion rates in Treatments 2 and 3 . All diets were devoid of feed antibiotics and pharmaceutical levels of zinc and copper. Pigs were fed a three-phase regimen, with a 14-day duration per phase. Pigs' body weight, feed intake, and fecal samples were collected at the end of each phase. Blood samples were collected to determine plasma lactylates and complete blood cell count.

\subsection{Microbial Composition in Nursery Pigs}

A total of 172 rectal swabs passed the quality and rarefying procedures, yielding a total of 1,028,904 high-quality reads clustered into 2438 features. These features were classified into 20 phyla and 182 genera (Figure 2a; Figure S1). Throughout the entire nursery stage, Firmicutes, Bacteroidetes, and Proteobacteria consistently represented the top three most dominant phyla, contributing $52 \%, 30 \%$, and $3.8 \%$ to overall phyla, respectively. There is an obvious transit of the microbial community at the genus level from weaning to nursery pigs. Prevotella is the most dominant genus member at weaning, and the dominance continues to increase during the nursery phase, maintaining the highest abundance among all taxa. Similar to Prevotella, genera Lactobacillus, Ruminococcaceae, Megasphaera, and 
Blautia increased rapidly in the first two weeks after weaning, emerging as other dominant members in the nursery phase (Figure S1). Some taxa such as Bacteroides, Fusobacterium, and Mogibacterium, occupying $1.8 \sim 6 \%$ of total taxa at weaning, were adversely affected regarding their abundances $(<0.005 \%)$ at the end of Phase 1 (Figure S1). A similar pattern was observed at the feature level; nine Prevotella features and three Lactobacillus features were enriched greatly on the first observation day, although to a different extent (Figure 2a).
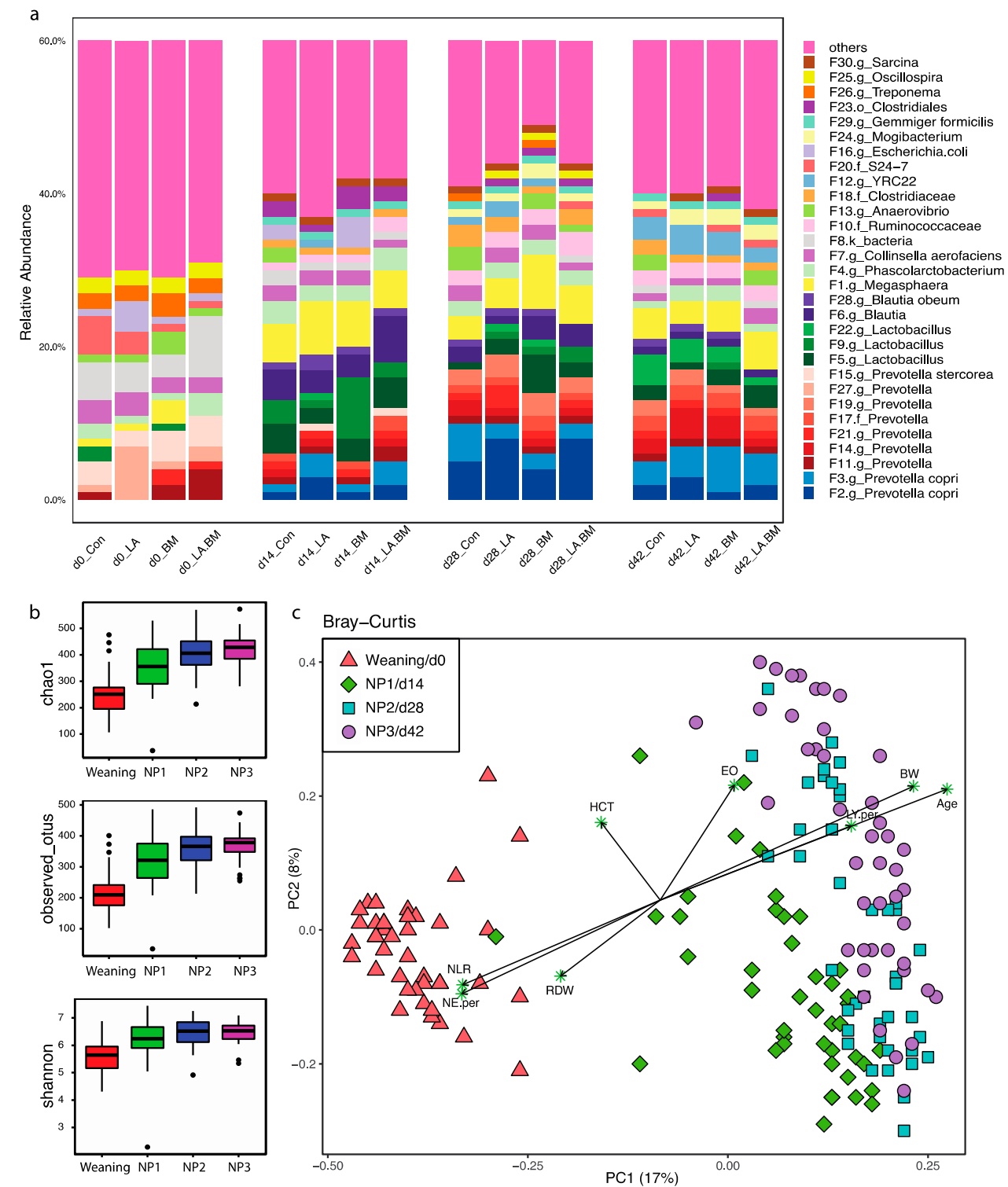

Figure 2. Characterizations of gut microbiome compositions (a) and community dynamics (b) and alpha diversity and (c) beta diversity at weaning (d0) and the end of each nursery phase (NP): NP1 (d14), NP2 (d28), and NP3 (d42). Rectal swabs were collected from all four treatments at the end of each phase and subjected to DNA extraction and 16S rDNA amplicon sequencing. Mothur software v.1.39.5 was used to calculate the coordinates for each significantly affected blood/bacterial component (EO, eosinophil; HCT, hematocrit; NLR, neutrophil-lymphocyte ratio; RDW, red cell distribution width; LY.per, percentage of lymphocyte).

\subsection{Effect of Age on Gut Microbial Structures and CBC Profiles}

Microbiota diversity and richness steadily increased throughout the nursery stage, as indicated by the increasing alpha indexes of Chao1, observed features, and Shannon (Figure 2b). During the first two weeks, the communities demonstrated the greatest 
diversity change among all three time intervals, after which the change slowed until the end of the study. As shown in the principal coordinate analysis (PCoA) plot based on BrayCurtis distances, community structures of pigs on weaning day were significantly different from those of older nursery pigs (Figure 2c). However, despite being distinguishable, Phase 1 , Phase 2, and Phase 3 microbiota communities had greater similarities than differences when compared to weaning day observations. These observations were in line with both alpha diversity and compositional changes during the crucial transition from a sow milk diet to solid feed (Figure 2c). By aligning the Bray-Curtis-distance-based coordinates with significantly changed blood cell counts and phenotypes, neutrophil-lymphocyte ratio (NLR), NE\%, and red cell distribution width (RDW) were more correlated with gut microbiomes in weaning pigs fed sow milk, while EO and lymphocyte percentages were more associated with those in nursery pigs fed a solid diet. This relationship was enhanced in the older-age nursery pigs (Figure 2c).

To identify the different bacterial features within the four time points, feature tables (top 300 features) from all samples were included for linear discriminant analysis effect size (LEfSe, Figure 3a,b). A total of 31 features were detected to be day-associated. Specifically, F24 Mogibacterium, F164 Desulfovibrio, F150 Mogibacteriaceae_unclassified, and F52 Prevotella tended to be highly presented in weaning pigs. Five features, including F6 Blautia, F60 Lactobacillus, and F128 Coprococcus, were enriched at the end of NP1 (D14). Four features (F175/F102 Prevotella, F137 Turicibacter, and F240 02d06) were NP2-associated (D28). At the end of NP3, when the pigs were two months old, a greater number of microbial features were discovered at a higher abundance, which included four Prevotella (F14, F17, F39, and F190), three Ruminococcaceae_unclassified (F193, F158, and F212), and two Veillonellaceae_unclassified (F57 and F107; Figure 3a,b).
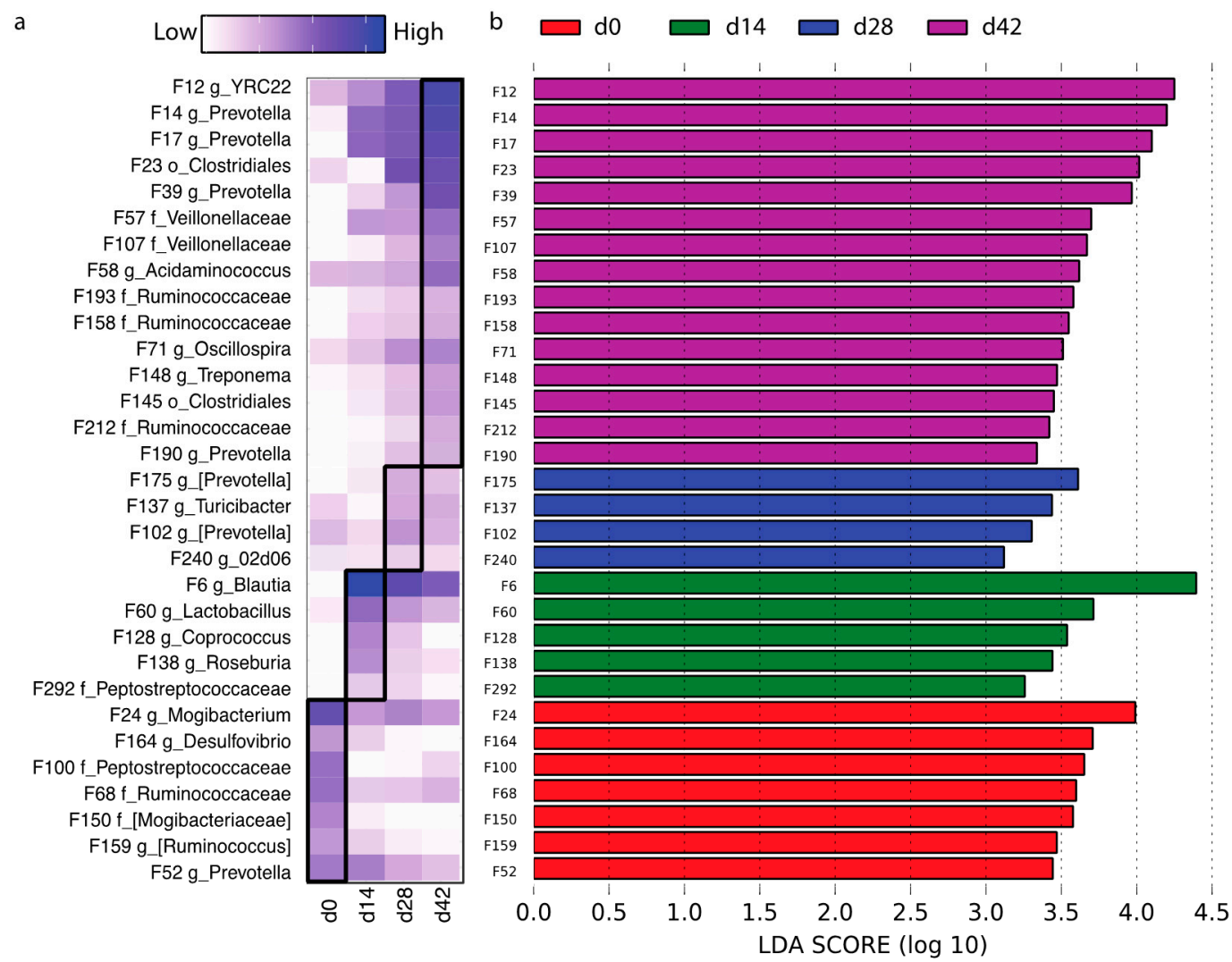

Figure 3. Abundances of age-associated bacterial features at weaning (d0) and the end of each nursery phase: d14, d28, and d42 (a). Discrepant bacterial members between groups were determined using online software LEfSe (b) (https: //huttenhower.sph.harvard.edu/galaxy/; access date: 1 December 2020). 


\subsection{Effects of Dietary Lactylate and Bacillus Subtilis on the Microbiota Community in Nursery Pigs}

Lactylate and Bacillus subtilis had a crucial impact on growth performance. Diet is one of the main drivers of gut microbiome structure. Studying the altered microbial taxa in the gut can provide a better understanding of the principles leading to beneficial effects. In this section, we investigated how dietary supplements (lactylates or Bacillus subtilis) modulated overall community structure and individual bacterial taxa members.

Pigs that received dietary lactylate (Treatment 2 (2.LA) and Treatment $4(4 . L A+B M)$ ) had improved ADG at Phase 1 (D14; Figure 1b). Next, we studied how the treatments affected their microbiota community shifts. Generally, adding lactylate (2.LA) in the diet increased richness at the genus level when compared to pigs fed control diets, as indicated by observed genus and Chao1 (Figure 4a). No significant change was observed for community diversity (Shannon) between the 1.Con and 2.LA groups. Interestingly, none of the alpha indexes were affected by supplementing both lactylates and Bacillus subtilis in the diet (4.LA+BM; Figure 4b). Beta-diversity based on Bray-Curtis dissimilarities presented distinguishable separations for both comparisons: 1.Con vs. 2.LA and 1.Con vs. 4.LA+BM (Figure 4c,d). To further reveal the altered bacterial members by treatments, the top 300 bacterial features from both groups were used for LEfSe analysis. In the comparison between 1.Con and 2.LA, only three bacterial members (F16 E. coli, F130 Eubacterium, and F555 Ruminococcaceae) were enriched in the control group, while ten members (such as F12 YRC22, F267 Christensenellaceae, F191 Succinivibrio, and F192 Clostridials_unclassified) were exclusively higher in abundance in the 2.LA treatment (Figure 4e). When comparing 4.LA+BM with the 1.Con groups, the relative abundances of F11 Prevotella, F192 Clostridials_unclassified, F189 Coriobacteriaceae, F79 Treponema, F492 Ruminococcaceae, and F248 Bacteroidales increased by the additional supplementation of lactylates and Bacillus subtilis, while F555 Ruminococcaceae, F16 E. coli, F46 Roseburia faecis, and F84 [Prevotella] had decreased relative abundance (Figure 4f). Overall, F16, F555, and F192 revealed a similar pattern in both comparative groups. These changes were possibly driven by dietary lactylate. It is worthy to note that the most abundant $E$. coli (F16) was consistently present in weanling pigs of all groups, from $1 \%$ to $4 \%$, while adding lactylate in the diet reduced the amount of F16 E. coli to under the minimum detectable level at the end of Phase 1: 1. Con $-2 \%, 2 . \mathrm{LA}-0 \%, 3 . \mathrm{BM}-4 \%$, and $4 . \mathrm{LA}+\mathrm{BM}-0 \%$ (Figure 2a).

We continued to analyze how feed-efficiency-associated Bacillus subtilis modulated microbial structures at the end of Phase 2 (D28). Similar or slightly reduced alpha diversities (observed_genus, Chao1, and Shannon) were observed in 2.BM and 4.LA+BM groups compared with the Con group separately (Figure S2a,b). PCoA based on Bray-Curtis distances disclosed differences in microbiota structures between BM or BM-devoid diets (Figure S2c,d). Bacterial features such as F1 Megasphaera, F112 Coprococcus, F189 Coriobacteriaceae_unclassified, F207 Gemmiger, and F326 [Prevotella] increased in pigs fed a diet supplemented with only BM (3.BM), while relative abundances of F564 Ruminococcus, F171 Dialister, F207 Gemmiger, F143 Succinivibrio, and certain others decreased (Figure S2e). Adding both LA + BM to the nursery diet increased F29 Gemmiger formicilis, F112 Coprococcus, F39 Prevotella, F591 Mitsuokella, and F395/F410 Ruminococcaceae in the gut at NP2 but decreased the abundances of F135 Prevotella, F584 Bacteroides, F143 Succinivibrio, F34 Prevotella, and F20 S24-7 (Figure S2f). Overall, the Bacillus subtilis treatment enriched F112 and reduced F20 and F143 in pigs at the end of NP2. 

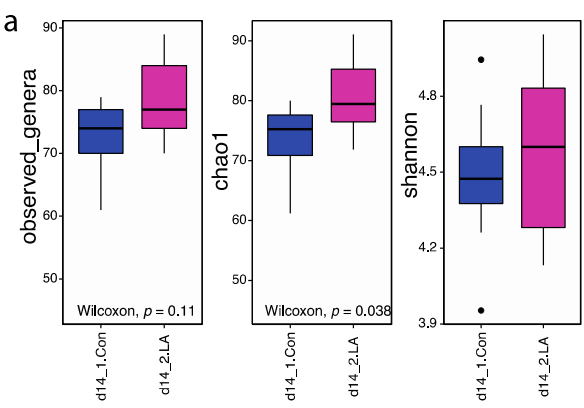

C Bray-Curtis NP1_d14

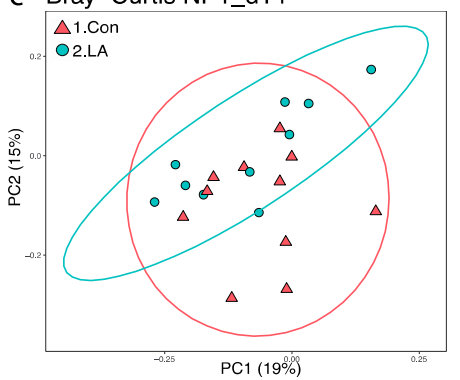

e
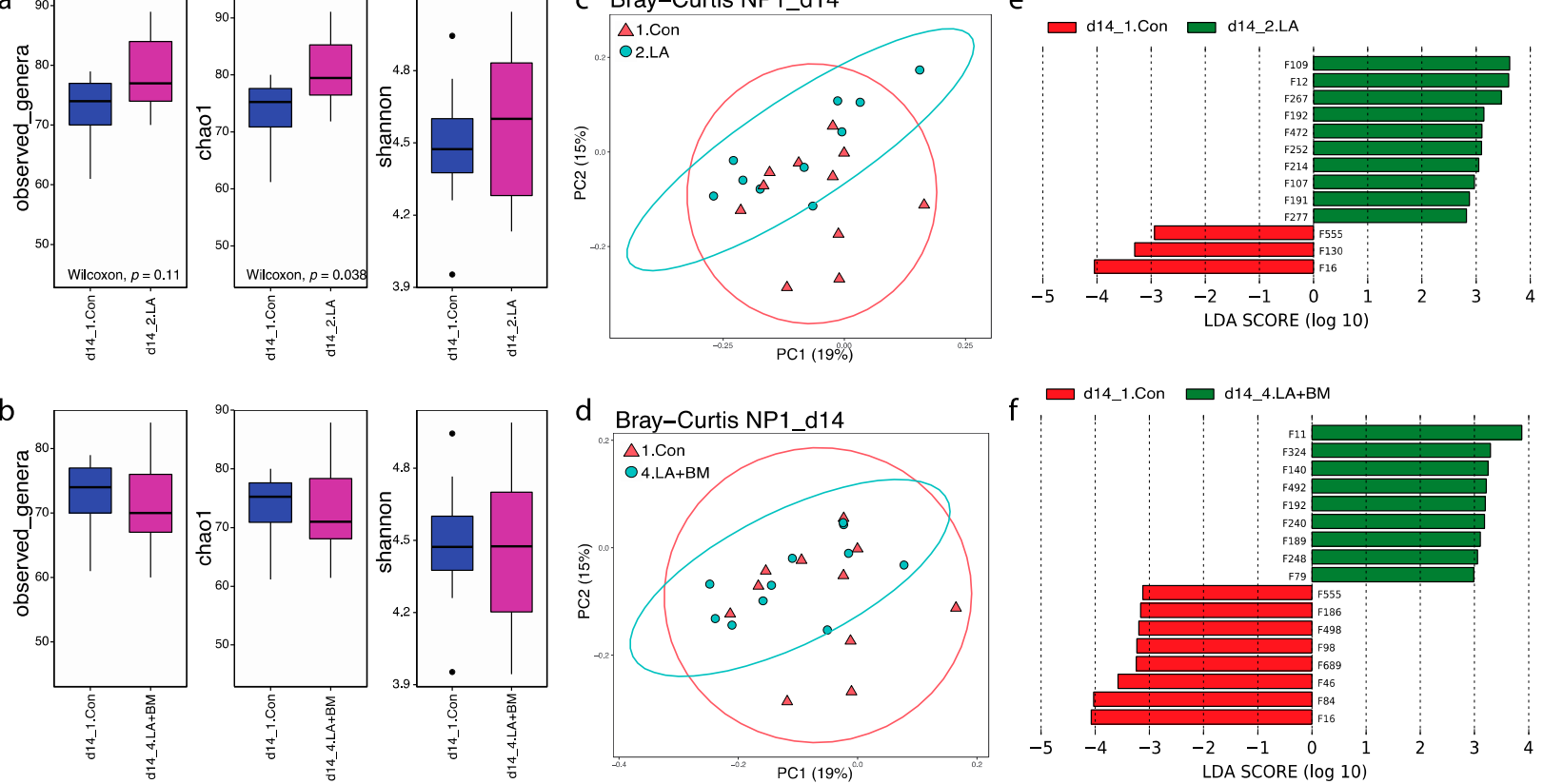

Figure 4. Dietary lactylate (2.LA and 4. LA+BM) modulated gut microbiota (alpha diversities: (a,b); beta diversities: (c,d)) and single bacterial members (e,f) during the first phase after weaning. Dietary lactylate increased microbial richness, and Bray-Curtis distances show a distinguished separation between the two groups. LEfSe disclosed the features with significant changes when adding lactylate to the diet.

\subsection{Feed Efficiency and ADG-Associated Bacterial Features}

We observed improved feed intake and average daily gain in pigs fed lactylates during Phase 1 and improved feed efficiency in pigs fed the Bacillus subtilis mixture during Phases 1 and 2 . These phenotypes were likely enhanced by gut microbiota compositions due to their main roles in fiber digestion and essential nutrient production. We next constructed a regression-based random forest on these observations with the top 400 bacterial features to identify ADG- or FE-associated beneficial taxa. At the end of NP1, F363, F191, F11, F60, and so forth were associated with ADG, while F11, F363, F191, F470, F186 (Figure 5a), and so forth were associated with FE (Figure 5b). Among these features, we observed that F11 Prevotella (Figure 5c,e) and F191 Succinivibrio (Figure 5d,f) presented positive correlations with both ADG and FE. Moreover, F191 increased when adding lactylates (Figure 5h), and F11 increased in diets supplemented with Bacillus subtilis or lactylates alone, with diets containing both BM and LA having the highest level (Figure 5g). At the end of NP2, F390, F9, F196, F269, F480, F290, and certain others were the top-most FE-associated taxa; F198 and F141 possessed positive relationships with FE, and both were enriched by the Bacillus subtilis treatment (Figure S3). 

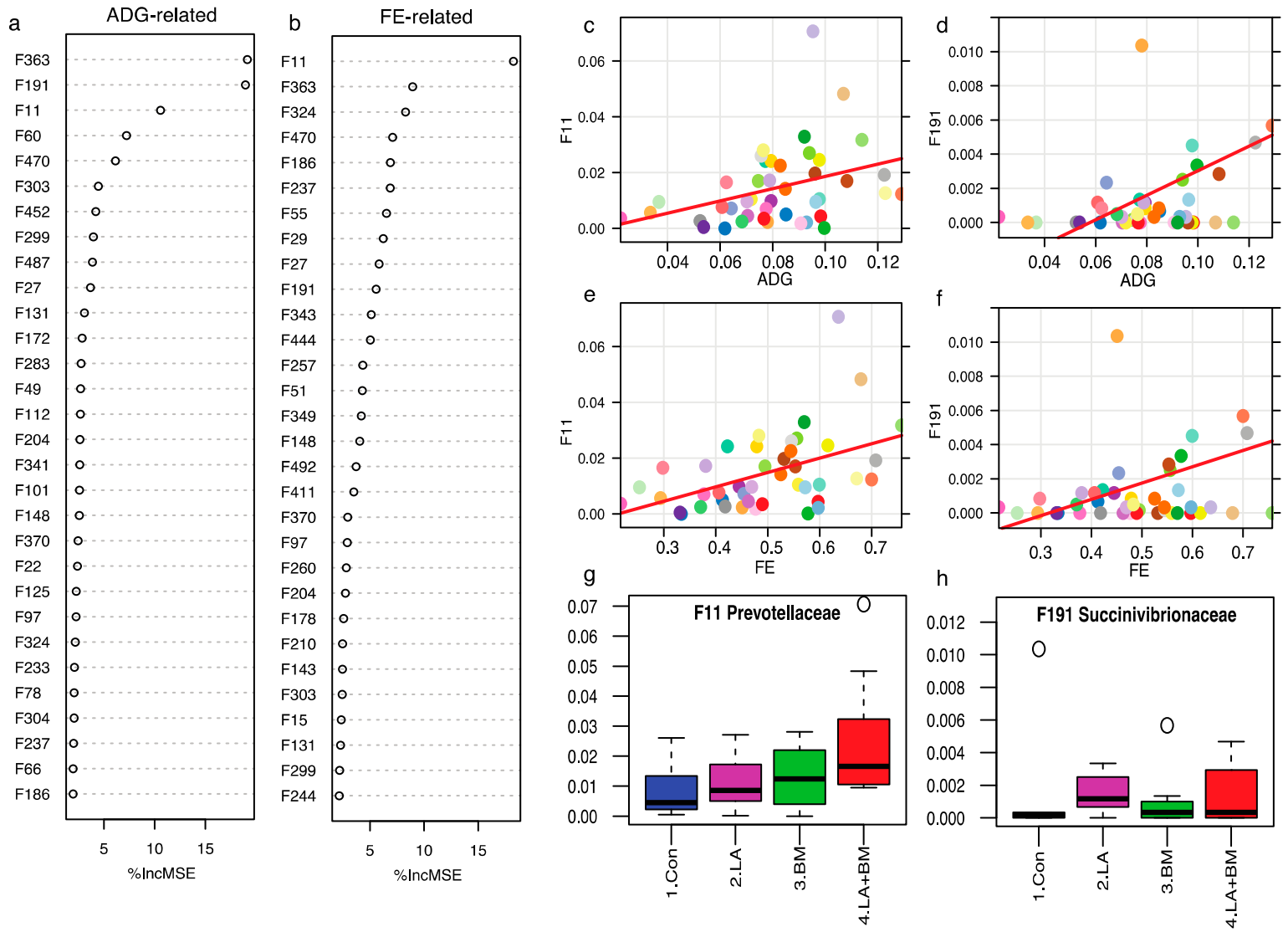

Figure 5. Regression-based random forest disclosed ADG-(a) and feed efficiency (FE)-associated (b) features (Top 30) at the end of nursery phase 1 (NP1). Bacterial features F11 and F191 were positively related to ADG (c,e, respectively) and FE (d,f, respectively). Additional lactylates in the diet can enrich both features at NP1 (g,h, respectively).

\section{Discussion}

\subsection{Lactylate and Bacillus subtilis Effects on Phenotypes}

Dietary intervention has become a very important focus within the swine industry to help minimize the detrimental effects of weaning $[10,19,29,30]$. The current study characterized the capabilities of probiotics and lactylates on growth performance, feed efficiency, peripheral blood cell counts, and gut microbiota changes during the nursery stage.

Piglets fed a lactylate-supplemented diet containing esters of lactic acid and various medium-chain fatty acid (lauric and myristic acid) compounds resulted in increased ADG and ADFI in the first two weeks postweaning. Our results are in line with previously published studies where piglets fed with $0.1 \%$ sodium stearoyl-2-lactylate in the diet significantly increased ADG and ADFI during the first 17 days [15]. An increased feed intake postweaning indicates a healthier gut environment physically and histologically. However, no significant growth-promoting effects were observed for the remaining trial period, which was similar to our results: a significantly improved ADG was only observed during the first two weeks of lactylate application. Hence, lactylates could be supplemented in the early postweaning diet as transient pacifiers to minimize weaning stress and to reduce the incidences of disease, difficulty of management, and economic cost. In our study, Bacillus subtilis, a mixture of two Bacillus subtilis strains, significantly increased feed efficiency during the early nursery stage. Interestingly, there was a synergistic improvement in feed efficiency when lactylate and Bacillus subtilis were both added to the diet during the first four weeks. The feed conversion ratio is one of the major parameters assessing economic value for the swine industry; thus, Bacillus subtilis could represent a feed efficiency 
booster and have potential benefits to growth performance, gut health, tight junctions, immune response, and the gut microbiome [20-23].

The circulatory system is the carrier for the cycling of oxygen, nutrients, wastes, and other functional molecules [31]. It also assists the immune system by transporting cells between the central and peripheral lymphoid organs. Eosinophils are immunerelated white blood cells that can help defend the host by releasing toxic compounds against invading pathogens [32]. It has been reported that an increase in circulating eosinophils is associated with stimulated intestinal tissue repair function when infection occurs $[33,34]$. In contrast, there was a greater decline of peripheral eosinophil percentage in pigs fed lactylate at the end of Phase 1, while other phases remained consistent. This potentially indicated that those pigs were situated under a healthier status compared to their counterparts. A similar pattern was observed in feed intake and enterotoxigenic E.coli count. Caloric intake is suggested to promote the homing of eosinophils to small intestine tissue via the activation of type II innate lymphoid cells [35]. Therefore, higher feed intake in Phase 1 could be the cause of the declining percentage of eosinophil in pigs fed supplemented lactylate. Moreover, the reduction in ETEC count indicates a lower risk of infection, and increased intake contributes to improved growth performance rather than tissue repairing. To further explain the relationships with gut integrity, measures of eosinophils and tight junction proteins in the local tissues are required. Hemoglobin counts and hematocrit\% are parameters of risk for anemia in weaning pigs. Piglets with a hemoglobin concentration lower than $9.0 \mathrm{~g} / \mathrm{dL}$ are classified as anemic [36]. Hence, lactylate could reduce the incidence of anemia in weaned pigs by increasing hemoglobin and hematocrit $\%$ [37].

One cause of high morbidity and mortality in postweaning pigs is their high susceptibility to pathogens. We quantified the populations of E. coli and ETEC in their feces by the spread plate technique and observed a significant reduction in their numbers, which correlated with the addition of dietary lactylate. This suggested that lactylate could be a potential replacement for antibiotics to help protect weaned pigs by reducing pathogen abundance.

\subsection{Longitudinal Gut Microbiome Dynamics}

We utilized the $16 \mathrm{~S}$ rDNA amplicon sequencing technique and observed gut microbiome transformations from weaning to the end of the nursery stage, which involved two very different living conditions. The 20-day-old piglets had to endure substantial stressors mainly from two aspects: (1) from an easily digestible sow milk diet to less-digestible solid feed and (2) from a sow/litter isolated facility to a crowded nursery where pen mates are a mix of different litters. The abrupt physical and dietary changes, generating considerable stress, can cause digestion reconstruction and immune system disruption, which can lead to impaired gut integrity and dysbiosis [7]. Correspondingly, the gut microbiota community undergoes reestablishment by peer competition and environmental selection. From weaning to NP1, there were significant differences in microbiota composition due to various available fiber sources and other undigested compounds in the distal gut. For instance, Prevotella copri was observed to develop rapidly during this period due to the increased plant fiber in the diet [38]. Their relative abundance increased during NP2 and then slightly decreased in NP3. Lactobacillus (F5, F9, and F22), Blautia (F6 and F28), and Megasphaera (F1) were three taxa that multiplied considerably during NP1; they are known to consume dietary fiber [39]. In addition, various appearing preferences were also discovered for bacteria under the same genus classification: Prevotella (F15 and F27) increased in weaning pigs consuming sow milk, while other Prevotella members increased with the feeding of a solid diet. However, we discovered that taxa from the genus Fusobacterium were significantly reduced from weaning $3.6 \%$ to NP1 0.01\% (Figure S1), which agrees with a previously published study [40]. In addition, Fusobacterium was reported to be dominant in preweaning pigs, and their abundance was higher in piglets with diarrhea than either 
healthy pigs or those receiving a practical creep-feed during the lactation stage [41]. Hence, diet type might cause the dramatic drop in Fusobacterium postweaning [42].

Besides diet type as the major influencer on gut microbiota, aging, with its physical and histological changes, could also selectively influence the microbiota. From NP1 to $\mathrm{NP3}$, even with similar formulated diets, continuous internal community readjustment was observed. For instance, F6 Blautia peaked at NP1 and then experienced continuous reduction until the end of the nursery stage. F12 YRC22 slowly increased its abundance from NP1 and reached the highest abundance in NP3 (Figure 2a). These observations may have resulted from the maturation of the gut [40].

\subsection{Lactylate and Bacillus subtilis Modulated Gut Microbiome}

Gut microbiota executes multiple tasks, including food digestion, nutrient exchange, immune response, and signal transduction between the central and enteric nervous systems [43]; thus, subtle changes in the microbiota may, to some extent, lead to different physiological changes. In the current study, we provide evidence supporting how the diet intervention of lactylate and Bacillus subtilis modulated the gut microbiota structure. Similar to the short-term effects on ADG, pigs fed with dietary lactylate had greater microbial richness in NP1 compared to the Con group. Distinguishable microbiome communities were also observed. However, pigs that received additional Bacillus subtilis supplementation (LA + BM group) had the highest ADG among the four treatments but had unchanged community richness and diversity. This indicates that increased gut microbiota diversity and total observed taxa members might not be prerequisites for potential beneficial effects. The same phenomenon was observed with improved feed efficiency in pigs fed LA + BM in NP2: neither adding Bacillus subtilis nor a combination of lactylate and Bacillus subtilis increased gut community richness and diversity.

Interestingly, LEfSe analysis revealed significantly modified taxa and introduced a better understanding of how dietary interventions can enhance the health of pigs.

First, lactylate or the LA + BM combination in the diet effectively reduced the relative abundances of F16 E. coli in the piglets in Phase 1, which has also been validated by the culture-dependent quantification method, as previously described. E. coli or ETEC are major pathogens that invade during lactation and the postweaning period, causing severe dysbiosis, increased intestinal permeability, diarrhea, and reduced growth performance [23,44]. Therefore, lactylate can help protect the piglets from E. coli infection.

Second, supplementing lactylate to weaned pigs boosted the relative abundances of beneficial bacteria in the gut, such as F11 Prevotella, F267 Christensenellaceae, F191 Succinivibrio, and F192 Clostridiales_unclassified. These community members have been frequently discussed for their important functions in young pigs. Members of Cristensenellaceae are involved in protein- and plant-sourced fiber fermentation. In other studies, members of this family have been determined to be feed-efficiency-associated bacteria [45-47]. F192, an unclassified Clostridiales was stimulated in both 2.LA and 4.LA+BM groups. Clostridiales have been reported to possess multiple salutary functions. Some species can attenuate severe inflammatory responses, while others can enhance intestinal barrier functions through the production of butyrate and indolepropoinic acid that help to fuel the epithelium [48]. It is noteworthy to mention that F11 and F191 were regressionbased random-forest-selected growth-performance- and feed-efficiency-associated bacterial members. F191 Succinivibrio is a core microbiota member in swine and is involved in carbohydrate metabolism, resulting in the production of acetate and succinate.

Third, lactylate expedites gut microbiota development during the first two weeks of weaning. As discussed by previous longitudinal studies, age and diet type are two major factors causing microbiota shifts [49,50]. Early gut microbiota development follows a certain specific course in healthy pigs, which is defined as microbiota maturation [28,42]. Generally, healthy pigs are less susceptible to a stressful environment and can rapidly restore their gut microbiota during the weaning to nursery transition. In our study, Prevotella gradually increased until the end of NP3 and thus, could be used as an indicator 
of age or gut microbiota maturation during the nursery stage. In NP1, abundant bacterial features from Prevotella copri, Prevotella (Figure 2a), and the genus Prevotella (Figure S1) were stimulated by dietary lactylate, which indicated a faster maturation process. Overall, our results suggest that lactylate can be used, through oral administration, to stabilize the gut microbiota community and improve growth performance.

Bacillus subtilis inclusion in the diet had no impact on feed intake but improved the feed conversion ratio. Hence, it significantly stimulated the efficacies of nutrient digestion and metabolism. Using regression-based random forest, we found abundances of F198 S24-7_unclassified and F141 f_Ruminococcaceae_unclassified, which were positively related to feed efficiency and were stimulated in piglets fed Bacillus subtilis. Similarly, taxa from Ruminococcaceae were classified as feed-efficiency-promoting members, as previously indicated [51]. On the other hand, F198 S24-7_unclassified was discovered as a predominant genus in pigs with a low feed efficiency [47]. However, another much more abundant feature, F20 S24-7 (ten times F198), demonstrated a decline in the Bacillus subtilis groups. F112 Coprococcus, one of the major butyrate producers, was also stimulated by Bacillus subtilis [52]. Overall, dietary Bacillus strains enriched the bacteria with high fiber fermentation efficacy, which may enhance their potential as a source of prospective DFM.

In conclusion, the current study evaluated the effects of both lactylate and Bacillus subtilis on growth indexes, blood cell profiles, and microbiota shifts in nursery pigs. Lactylate exhibited beneficial promoting effects on average daily gain during the first two weeks of the nursery stage. This response can be explained, in part, by the increasing levels of neutrophils in systemic circulation and the decreasing fecal E. coli and ETEC counts. The addition of lactylate in the diet increased the overall microbiome richness and maturity and modulated the gut microbiome by reducing F16 E. coli and increasing the beneficial bacterial features of F267 Christensenellaceae, F191 Succinivibrio, and F192 Clostridiales_unclassified. Pigs fed Bacillus subtilis had enriched high feed-efficiency-associated features (F141 f_Ruminococcaceae and F198 S24-7_unclassified) and butyrate-producing taxa (F122 Coprococcus). The combination of lactylate and Bacillus subtilis strains showed the greatest feed efficiency and increased the top-most feed-efficiency-associated taxa (F11 Prevotella) among treatments and further reduced total E. coli and enterotoxigenic E. coli counts from pigs fed lactylate alone. Collectively, these findings suggested that a synergistic effect was exerted by the lactylate and Bacillus subtilis mixture, and this combination could be added to diets to balance gut microbiota and improve growth performance during the early postweaning period.

Supplementary Materials: The following are available online at https: / www.mdpi.com/article/ 10.3390 / microorganisms 9040803 /s1, Figure S1: Characterization of gut microbiome compositions at the phylum and genus levels during the nursery stage. Rectal swabs were collected from all four treatments at the end of each phase and subjected to DNA extraction and 16S rDNA amplicon sequencing; Figure S2. Dietary Bacillus subtilis mixture (3.BM and 4. LA+BM) modulated the overall gut microbiome communities (alpha diversities: $a$ and $b$; beta diversities: $c$ and $d$ ) and single bacteria member (e and f) during the second phase after weaning. Dietary lactylate increased the microbial richness, and Bray-Curtis distances show a distinguishable separation between the two groups. LEfSe disclosed features with significant changes by adding the Bacillus subtilis mixture to the diet; Figure S3. Regression-based Random Forest disclosed ADG associated features (top 30) at the end of phase 2. Bacterial features F141 and F198 were positively related to FE. Adding the Bacillus subtilis mixture to the diet can enrich both features in phase 2; Table S1: Control diet composition (As fed)1; Table S2. Plasma C12 (lauric acid) and C14 (myristic acid) lactylate level (means \pm sem); Table S3. Main effect of adding lactylates (LA) and/or Bacillus subtilis mixture (BM) on growth performance of nursery pigs (LS means); Table S4. Lactylates and Bacillus subtilis mixture interaction effect on growth performance of nursery pigs (LS means); Table S5 lactylate and age interaction effect on peripheral complete blood cell count (LS means); Table S6 Bacillus subtilis mixture and age interaction effect on peripheral complete blood cell count (LS means); Table S7. Fecal microbial counts and prevalence obtained at baseline (Phase 0 ) and at the end of each nursery feed phase. 
Author Contributions: E.D. and T.T. conceived and designed this experiment. X.W. (Xiaoyuan Wei), B.Z., and T.T. collected rectal samples and growth performance data. T.R. and S.H. contributed to part of the materials and methods of the manuscript and performed the microbial counts. X.W. (Xiaofan Wang) and T.T. analyzed the data. X.W. (Xiaofan Wang), T.T., and J.Z. drafted and revised the manuscript with input from the other coauthors. C.V.M. conceptualization, Supervision and Writing-review editing. E.J.M.J. Methodology. All authors have read and agreed to the published version of the manuscript.

\section{Funding: None.}

Institutional Review Board Statement: The study was conducted according to the guidelines of the University of Arkansas Institutional Animal Care and Use Committee (IACUC\# 21038, 4/20/20).

Data Availability Statement: Data are available on the National Center for Biotechnology Information (NCBI) Short Read Archive database (SUB9202712). The BioProject accession number is PRJNA706745.

Conflicts of Interest: The authors declare no conflict of interest.

\section{References}

1. Frese, S.A.; Parker, K.; Calvert, C.C.; Mills, D.A. Diet shapes the gut microbiome of pigs during nursing and weaning. Microbiome 2015, 3, 1-10. [CrossRef]

2. Wang, H.; Xu, R.; Zhang, H.; Su, Y.; Zhu, W. Swine gut microbiota and its interaction with host nutrient metabolism. Anim. Nutr. 2020, 6, 410-420. [CrossRef] [PubMed]

3. Guevarra, R.B.; Lee, J.H.; Lee, S.H.; Seok, M.-J.; Kim, D.W.; Kang, B.N.; Johnson, T.J.; Isaacson, R.E.; Kim, H.B. Piglet gut microbial shifts early in life: Causes and effects. J. Anim. Sci. Biotechnol. 2019, 10, 1-10. [CrossRef] [PubMed]

4. Collins, C.L.; Pluske, J.R.; Morrison, R.S.; McDonald, T.N.; Smits, R.J.; Henman, D.J.; Stensland, I.; Dunshea, F.R. Post-weaning and whole-of-life performance of pigs is determined by live weight at weaning and the complexity of the diet fed after weaning. Anim. Nutr. 2017, 3, 372-379. [CrossRef] [PubMed]

5. Cash, H.L.; Hooper, L.V. Commensal bacteria shape intestinal immune system development. ASM News 2005, $71,77-83$.

6. Everaert, N.; Van Cruchten, S.; Weström, B.; Bailey, M.; Van Ginneken, C.; Thymann, T.; Pieper, R. A review on early gut maturation and colonization in pigs, including biological and dietary factors affecting gut homeostasis. Anim. Feed Sci. Technol. 2017, 233, 89-103. [CrossRef]

7. Chong, C.Y.L.; Bloomfield, F.H.; O'Sullivan, J.M. Factors affecting gastrointestinal microbiome development in neonates. Nutrients 2018, 10, 274. [CrossRef] [PubMed]

8. Chung, H.; Pamp, S.J.; Hill, J.A.; Surana, N.K.; Edelman, S.M.; Troy, E.B.; Reading, N.C.; Villablanca, E.J.; Wang, S.; Mora, J.R. Gut immune maturation depends on colonization with a host-specific microbiota. Cell 2012, 149, 1578-1593. [CrossRef]

9. Velayudhan, D.; Kim, I.; Nyachoti, C. Characterization of dietary energy in swine feed and feed ingredients: A review of recent research results. Asian-Australas. J. Anim. Sci. 2015, 28, 1. [CrossRef] [PubMed]

10. Wei, X.; Tsai, T.; Knapp, J.; Bottoms, K.; Deng, F.; Story, R.; Maxwell, C.; Zhao, J. ZnO modulates swine gut microbiota and improves growth performance of nursery pigs when combined with peptide cocktail. Microorganisms 2020, 8, 146. [CrossRef] [PubMed]

11. Nie, Y.-f.; Hu, J.; Yan, X.-h. Cross-talk between bile acids and intestinal microbiota in host metabolism and health. J. Zhejiang Univ. Sci. B 2015, 16, 436-446. [CrossRef]

12. Frobish, L.; Hays, V.; Speer, V.; Ewan, R. Effect of diet form and emulsifying agents on fat utilization by young pigs. J. Anim. Sci. 1969, 29, 320-324. [CrossRef]

13. Yun, H.; Yun, K.; Upadhaya, S.; Kim, I. Effect of supplementation of sodium stearoyl-2-lactylate as fat emulsifier in low-density diet on growth performance, backfat thickness, lean muscle percentage, and meat quality in finishing pigs. Can. J. Anim. Sci. 2018, 99, 132-137. [CrossRef]

14. Lee, S.J.; Lee, S.S.; Kim, E.T.; Jeong, J.S.; Lee, J.H.; Jeong, J.; Park, J.K.; Park, B.Y.; Jeong, H.Y.; Ki, K.S. Effect of optimal sodium stearoyl-2-lactylate supplementation on growth performance and blood and carcass characteristics in Hanwoo steers during the early fattening period. Asian-Australas. J. Anim. Sci. 2018, 31, 1442. [CrossRef]

15. Bai, G.; He, W.; Yang, Z.; Fu, H.; Qiu, S.; Gao, F.; Shi, B. Effects of different emulsifiers on growth performance, nutrient digestibility, and digestive enzyme activity in weanling pigs. J. Anim. Sci. 2019, 97, 4235-4241. [CrossRef]

16. Wang, C.; Bai, Y.; Zhao, X.; Shi, B.; Meng, X.; Shan, A. Effects of feeding sodium stearoyl-2-lactylate diets to lactating sows on performance, digestibility of nutrients, composition, and fat globule size in milk. J. Anim. Sci. 2017, 95, 5091-5099. [CrossRef]

17. Cho, J.H.; Zhao, P.; Kim, I.H. Effects of emulsifier and multi-enzyme in different energy densitydiet on growth performance, blood profiles, and relative organ weight in broiler chickens. J. Agric. Sci. 2012, 4, 161. [CrossRef]

18. Ali, S.F.; Chao, W.; Xiaoli, W.; Jintian, H.; Mingfa, W.; El-Hack, M.E.A.; Lili, Z.; Xiang, Z.; Tian, W. Growth, serum biochemical indices, antioxidant status and meat quality of broiler chickens fed diet supplemented with sodium stearoyl-2 lactylate. Pak. Vet. J. 2017, 37, 445-449. 
19. Liu, Y.; Espinosa, C.D.; Abelilla, J.J.; Casas, G.A.; Lagos, L.V.; Lee, S.A.; Kwon, W.B.; Mathai, J.K.; Navarro, D.M.; Jaworski, N.W. Non-antibiotic feed additives in diets for pigs: A review. Anim. Nutr. 2018, 4, 113-125. [CrossRef] [PubMed]

20. Hu, Y.; Dun, Y.; Li, S.; Zhao, S.; Peng, N.; Liang, Y. Effects of Bacillus subtilis KN-42 on growth performance, diarrhea and faecal bacterial flora of weaned piglets. Asian-Australas. J. Anim. Sci. 2014, 27, 1131. [CrossRef] [PubMed]

21. Luise, D.; Bertocchi, M.; Motta, V.; Salvarani, C.; Bosi, P.; Luppi, A.; Fanelli, F.; Mazzoni, M.; Archetti, I.; Maiorano, G. Bacillus sp. probiotic supplementation diminish the Escherichia coli F4ac infection in susceptible weaned pigs by influencing the intestinal immune response, intestinal microbiota and blood metabolomics. J. Anim. Sci. Biotechnol. 2019, 10, 74. [CrossRef]

22. Zhang, W.; Zhu, Y.-H.; Zhou, D.; Wu, Q.; Song, D.; Dicksved, J.; Wang, J.-F. Oral administration of a select mixture of Bacillus probiotics affects the gut microbiota and goblet cell function following Escherichia coli challenge in newly weaned pigs of genotype MUC4 that are supposed to be enterotoxigenic E. coli F4ab/ac receptor negative. J Appl. Environ. Microbiol. 2017, 83. [CrossRef]

23. Kim, K.; He, Y.; Xiong, X.; Ehrlich, A.; Li, X.; Raybould, H.; Atwill, E.R.; Maga, E.A.; Jørgensen, J.; Liu, Y. Dietary supplementation of Bacillus subtilis influenced intestinal health of weaned pigs experimentally infected with a pathogenic E. coli. J. Anim. Sci. Biotechnol. 2019, 10, 52. [CrossRef]

24. Meer, R.; Songer, J.G. Multiplex polymerase chain reaction assay for genotyping Clostridium perfringens. Am. J. Vet. Res. 1997, $58,702-705$.

25. Stacy-Phipps, S.; Mecca, J.J.; Weiss, J.B. Multiplex PCR assay and simple preparation method for stool specimens detect enterotoxigenic Escherichia coli DNA during course of infection. J. Clin. Microbiol. 1995, 33, 1054-1059. [CrossRef] [PubMed]

26. Casey, T.A.; Bosworth, B.T. Design and evaluation of a multiplex polymerase chain reaction assay for the simultaneous identification of genes for nine different virulence factors associated with Escherichia coli that cause diarrhea and edema disease in swine. J. Vet. Diagn. Investig. 2009, 21, 25-30. [CrossRef]

27. Okwumabua, O.; O'Connor, M.; Shull, E. A polymerase chain reaction (PCR) assay specific for Streptococcus suis based on the gene encoding the glutamate dehydrogenase. FEMS Microbiol. Lett. 2003, 218, 79-84. [CrossRef]

28. Wang, X.; Tsai, T.; Deng, F.; Wei, X.; Chai, J.; Knapp, J.; Apple, J.; Maxwell, C.V.; Lee, J.A.; Li, Y. Longitudinal investigation of the swine gut microbiome from birth to market reveals stage and growth performance associated bacteria. Microbiome 2019, 7, 109. [CrossRef]

29. Bokulich, N.A.; Chung, J.; Battaglia, T.; Henderson, N.; Jay, M.; Li, H.; Lieber, A.D.; Wu, F.; Perez-Perez, G.I.; Chen, Y. Antibiotics, birth mode, and diet shape microbiome maturation during early life. Sci. Transl. Med. 2016, 8, 343ra382. [CrossRef]

30. Debski, B. Supplementation of pigs diet with zinc and copper as alternative to conventional antimicrobials. Pol. J. Vet. Sci. 2016, 19, 917-924. [CrossRef]

31. Zhu, A. Introduction to porcine red blood cells: Implications for xenotransfusion. Semin. Hematol. 2000, 37, 143-149. [CrossRef]

32. Wen, T.; Rothenberg, M.E. The regulatory function of eosinophils. J. Myeloid Cells Health Dis. Synth. 2017, 257-269. [CrossRef]

33. Hogan, S.P.; Waddell, A.; Fulkerson, P.C. Eosinophils in infection and intestinal immunity. Curr. Opin. Gastroenterol. 2013, $29,7$. [CrossRef]

34. Shah, K.; Ignacio, A.; McCoy, K.D.; Harris, N.L. The emerging roles of eosinophils in mucosal homeostasis. Mucosal Immunol. 2020, 13, 574-583. [CrossRef] [PubMed]

35. Travers, J.; Rothenberg, M. Eosinophils in mucosal immune responses. Mucosal Immunol. 2015, 8, 464-475. [CrossRef] [PubMed]

36. Bhattarai, S.; Nielsen, J.P. Early indicators of iron deficiency in large piglets at weaning. J. Swine Health Prod. $2015,23,10-17$.

37. Estienne, M.J.; Clark-Deener, S.G.; Williams, K.A. Growth performance and hematology characteristics in pigs treated with iron at birth and weaning and fed a nursery diet supplemented with a pharmacological level of zinc oxide. J. Swine Health Prod. 2019, $27,64-75$.

38. Fehlner-Peach, H.; Magnabosco, C.; Raghavan, V.; Scher, J.U.; Tett, A.; Cox, L.M.; Gottsegen, C.; Watters, A.; Wiltshire-Gordon, J.D.; Segata, N. Distinct polysaccharide growth profiles of human intestinal Prevotella copri isolates. BioRxiv 2019, 750802. [CrossRef]

39. Jefferson, A.; Adolphus, K. The effects of intact cereal grain fibers, including wheat bran on the gut microbiota composition of healthy adults: A systematic review. Front. Nutr. 2019, 6, 33. [CrossRef] [PubMed]

40. Chen, L.; Xu, Y.; Chen, X.; Fang, C.; Zhao, L.; Chen, F. The maturing development of gut microbiota in commercial piglets during the weaning transition. Front. Microbiol. 2017, 8, 1688. [CrossRef] [PubMed]

41. Tan, Z.; Dong, W.; Ding, Y.; Ding, X.; Zhang, Q.; Jiang, L. Changes in cecal microbiota community of suckling piglets infected with porcine epidemic diarrhea virus. PLoS ONE 2019, 14, e0219868. [CrossRef]

42. Yang, Q.; Huang, X.; Wang, P.; Yan, Z.; Sun, W.; Zhao, S.; Gun, S. Longitudinal development of the gut microbiota in healthy and diarrheic piglets induced by age-related dietary changes. MicrobiologyOpen 2019, 8, e923. [CrossRef] [PubMed]

43. Carabotti, M.; Scirocco, A.; Maselli, M.A.; Severi, C. The gut-brain axis: Interactions between enteric microbiota, central and enteric nervous systems. Ann. Gastroenterol. Q. Publ. Hell. Soc. Gastroenterol. 2015, 28, 203.

44. Matsumoto, H.; Miyagawa, M.; Takahashi, S.; Shima, R.; Oosumi, T. Improvement of the enterotoxigenic Escherichia coli infection model for post-weaning diarrhea by controlling for bacterial adhesion, pig breed and MUC4 genotype. Vet. Sci. 2020, 7, 106. [CrossRef] [PubMed]

45. McCormack, U.M.; Curião, T.; Buzoianu, S.G.; Prieto, M.L.; Ryan, T.; Varley, P.; Crispie, F.; Magowan, E.; Metzler-Zebeli, B.U.; Berry, D. Exploring a possible link between the intestinal microbiota and feed efficiency in pigs. Appl. Environ. Microbiol. 2017, 83. [CrossRef] [PubMed] 
46. Yang, H.; Huang, X.; Fang, S.; He, M.; Zhao, Y.; Wu, Z.; Yang, M.; Zhang, Z.; Chen, C.; Huang, L. Unraveling the fecal microbiota and metagenomic functional capacity associated with feed efficiency in pigs. Front. Microbiol. 2017, 8, 1555. [CrossRef] [PubMed]

47. Si, J.; Feng, L.; Gao, J.; Huang, Y.; Zhang, G.; Mo, J.; Zhu, S.; Qi, W.; Liang, J.; Lan, G. Evaluating the association between feed efficiency and the fecal microbiota of early-life Duroc pigs using 16S rRNA sequencing. AMB Express 2020, 10, 1-11. [CrossRef] [PubMed]

48. Guo, P.; Zhang, K.; Ma, X.; He, P. Clostridium species as probiotics: Potentials and challenges. J. Anim. Sci. Biotechnol. 2020, 11, 115. [CrossRef] [PubMed]

49. Li, Y.; Wang, X.; Wang, X.-q.; Wang, J.; Zhao, J. Life-long dynamics of the swine gut microbiome and their implications in probiotics development and food safety. Gut Microbes 2020, 11, 1824-1832. [CrossRef] [PubMed]

50. Yatsunenko, T.; Rey, F.E.; Manary, M.J.; Trehan, I.; Dominguez-Bello, M.G.; Contreras, M.; Magris, M.; Hidalgo, G.; Baldassano, R.N.; Anokhin, A.P. Human gut microbiome viewed across age and geography. Nature 2012, 486, 222-227. [CrossRef]

51. Bergamaschi, M.; Tiezzi, F.; Howard, J.; Huang, Y.J.; Gray, K.A.; Schillebeeckx, C.; McNulty, N.P.; Maltecca, C. Microbiome composition differences among breeds impact feed efficiency in swine. Microbiome 2020, 8, 110. [CrossRef] [PubMed]

52. Louis, P.; Flint, H. Diversity, metabolism and microbial ecology of butyrate-producing bacteria from the human large intestine. FEMS Microbiol. Lett. 2009, 294, 1-8. [CrossRef] [PubMed] 